\title{
Multizentrische Anwendungsbeobachtung zum Einsatz eines topischen Kombinationspräparates aus Mometasonfuroat 0,1\% und Salicylsäure 5\% bei der Behandlung von mittelschwerer bis schwerer Psoriasis ${ }^{1}$
}

\author{
Multicenter Observational Study of Mometasone Furoate $0.1 \%$ - Salicylic Acid $5 \%$ Ointment in Topical \\ Treatment of Psoriasis Patients
}

\author{
Autoren \\ S. Grieb ${ }^{1}$, B. Karmann², R. Zurmühlen², D. Bruch-Gerharz \\ Institute \\ Hautklinik des Universitätsklinikums Düsseldorf \\ Essex Pharma GmbH, München
}

Bibliografie

DOI 10.1055/s-0028-1119462

Online-Publikation: 13.3. 2009

Akt Dermatol 2009; 35:

175-179 (c) Georg Thieme Verlag KG Stuttgart · New York ISSN 0340-2541

Korrespondenzadresse

Prof. Dr. med. Daniela

Bruch-Gerharz

Universitäts-Hautklinik

Moorenstr. 5

40225 Düsseldorf

bruch-gerharz@med.uni-

duesseldorf.de

\section{Zusammenfassung \\ $\nabla$}

In der vorliegenden multizentrischen Anwendungsbeobachtung wurden Daten zur Wirksamkeit, Sicherheit und Verträglichkeit eines topischen Kombinationspräparates aus Mometasonfuroat $0,1 \%$ und Salicylsäure $5 \%{ }^{1}$ in der Behandlung von 3652 Patienten mit mittelschwerer bis schwerer Psoriasis erhoben. Nach einer durchschnittlichen Beobachtungsdauer von 4 Wochen zeigte sich ein Rückgang aller Symptome der Psoriasis. Der globale klinische Eindruck war bei über $87 \%$ der Patienten gut bis exzellent. Mehr

\section{Einleitung}

Mit einer Prävalenz von etwa $2 \%$ gehört die Psoriasis zu den häufigsten Hautkrankheiten des Menschen überhaupt, und ihre Behandlung stellt nach wie vor eine besondere Herausforderung an die moderne Medizin dar [1-3]. Bei vielen Psoriasis-Formen ist die topische Behandlung wegen der geringen Nebenwirkungen die Therapie der ersten Wahl [1]. Grundvoraussetzung einer wirksamen Lokaltherapie ist das Eindringen der Wirkstoffe in die Haut. Dies wird jedoch meist durch die starken Schuppenauflagerungen auf dem psoriatischen Entzündungsherd verhindert $[4,5]$. Die erste und entscheidende Maßnahme ist daher, zu Beginn und auch während der Therapie für eine ausreichende Entschuppung der Krankheitsherde zu sorgen und damit die Grundlage für eine effektive antientzündliche Behandlung der Psoriasis zu schaffen $[3,6]$. Eine in dieser Hinsicht wesentliche Neuentwicklung stellt ein in Deutschland seit Mai 2005 zugelassenes, topi-

\footnotetext{
${ }^{1}$ Das Kombinationspräparat aus Mometasonfuroat 0,1\% und Salicylsäure 5\% wurde als ELOSALIC ${ }^{\circledR}$ in Deutschland im Mai 2005 zur Behandlung der Psoriasis vulgaris zugelassen.
}

als $84 \%$ waren subjektiv zufrieden bis sehr zufrieden mit dem Therapeutikum. Daneben betrug die Inzidenz unerwünschter Ereignisse, die im Zusammenhang mit der Behandlung standen, weniger als 0,4\%. Die überwiegende Anzahl der gemeldeten unerwünschten Ereignisse war unspezifischer Natur und von milder Ausprägung. Neue, zum Zulassungszeitpunkt nicht bekannte Nebenwirkungen traten nicht auf. Die gute Wirksamkeit und hohe Akzeptanz des Kombinationspräparates bei geringer Nebenwirkungsrate lässt sich somit auch unter Praxisbedingungen belegen.

sches Kombinationspräparat dar, welches das nicht-fluorierte Glukokortikosteroid Mometasonfuroat $0,1 \%$ und $5 \%$ ige Salicylsäure in einer Salbengrundlage enthält. Hiermit kann gleichzeitig abschuppend und entzündungshemmend therapiert werden. Zudem ermöglicht die abschuppende Wirkung der Salicylsäure ein besseres Eindringen des topisch applizierten Glukokortikosteroids in die Haut. Die Überlegenheit der fixen Kombination von Mometasonfuroat und Salicylsäure im Vergleich mit den Einzelkomponenten sowie einem Standardpräparat konnte bereits in mehreren klinischen Studien eindrucksvoll belegt werden [7-9]. Die vorliegende Anwendungsbeobachtung hatte das Ziel, die Praktikabilität einer täglich ein- bis zweimaligen Behandlung der Psoriasis mit dem topischen Kombinationspräparat aus Mometasonfuroat $0,1 \%$ und Salicylsäure $5 \%$ unter besonderer Berücksichtigung der Wirksamkeit und Sicherheit an einem grossen Patientenkollektiv unter Praxisbedingungen zu überprüfen. Dabei dokumentierten von April 2005 bis November 2005 insgesamt 741 dermatologische Praxen und Krankenhäuser in Deutschland Behandlungsdaten von 3652 Psoriasis-Patienten. 


\section{Methodik}

$\nabla$

Mithilfe einer offenen, multizentrischen, nicht-vergleichenden, nicht-randomisierten Anwendungsbeobachtung gemäß $\S 67 \mathrm{Ab}-$ satz 6 des Arzneimittelgesetzes wurden Erkenntnisse bei der routinemäßigen, alltäglichen Anwendung des topischen Kombinationspräparates aus Mometasonfuroat 0,1\% und Salicylsäure $5 \%$ gesammelt. Zielparameter waren die Beurteilung des Behandlungserfolges der topischen Therapie sowie der Verträglichkeit und Compliance durch Patient und Arzt. Die Auswertung erfolgte mit den Methoden der beschreibenden Statistik. Die Ergebnisse der Verlaufsbeobachtung wurden mithilfe von Häufigkeitstabellen dargestellt.

Patientencharakteristika. Eingeschlossen wurden insgesamt 3652 Patienten mit nachgewiesener und dokumentierter mittelschwerer bis schwerer Psoriasis, bei denen nach ärztlichem Ermessen die Indikation zur Therapie mit dem topischen Kombinationspräparat aus Mometasonfuroat 0,1\% und Salicylsäure $5 \%$ gestellt wurde ( Tab. 1). Die Anwendungsbeobachtung erfolgte in 741 dermatologischen Praxen und Krankenhäusern in Deutschland, wobei der Beobachtungszeitraum im Mittel 4 Wochen betrug. Während dieser Zeit erfolgten durchschnittlich 4 Visiten pro Patient. Das Alter der beobachteten Patienten lag im Mittel bei 49,4 ( $\pm 16,0)$ Jahren. Darunter waren 50 Patienten $(1,4 \%)$ unter 18 Jahre und davon 6 Patienten unter 12 Jahre alt. $52,1 \%$ der Patienten waren weiblichen und 46,4\% männlichen Geschlechts (für 55 Patienten lag keine Information bezüglich des Geschlechts vor). Zur Beurteilung des Schweregrades der Erkrankung wurde der „Psoriasis Area and Severity Index“ (PASI) herangezogen. Dieser betrug zu Beginn der Behandlung im Durchschnitt 22,4 \pm 12,4. Dabei hatten 86,7\% der Patienten einen PASI-Score $>10$ und 6,1\% der Patienten einen PASI-Score $\leq 10$ als Anhaltspunkt für eine leichte Ausprägung der psoriatischen Hautveränderungen (für 7,3\% der Patienten lag keine Information vor). Nahezu alle Patienten zeigten die typischen klinischen Psoriasis-Symptome Erythem (3613 Patienten), Infiltration (3612 Patienten) und Schuppung (3613).

Vorbehandlung. Die für die Behandlung mit dem topischen Kombinationspräparat aus Mometasonfuroat 0,1\% und Salicylsäure 5\% vorgesehenen Areale waren bei 58,9\% der Patienten mit anderen Lokaltherapeutika vorbehandelt worden; 38,2\% der Patienten erhielten keinerlei Vorbehandlung. Bei 25,2\% der Patienten bestanden Begleiterkrankungen (Herz-Kreislauferkrankungen, Stoffwechselerkrankungen, Atemwegserkrankungen, u.a.), welche bei 18,8\% dieser Patienten medikamentös behandelt wurden.

Verlauf der Behandlung. Die Anwendung des topischen Kombinationspräparates aus Mometasonfuroat 0,1\% und Salicylsäure $5 \%$ erfolgte durch den Patienten entsprechend der handelsüblichen Gebrauchsinformation. Die Behandlung wurde bei $50,8 \%$ der Patienten $1 \times$ bzw. bei $48,4 \%$ der Patienten $2 \times$ täglich durchgeführt. Die Dauer der Behandlung lag im Mittel bei 4 Wochen (41,0 $\pm 23,3$ Tage). Zwischen Therapiebeginn und Abschlussuntersuchung lagen durchschnittlich 4 Visiten. Bei allen $3652 \mathrm{~Pa}-$ tienten (100\%) wurden im Rahmen der 1 . Visite demographische und klinische Daten erhoben ( $\bullet$ Tab. 1). Dabei wurden die verschiedenen Zielparameter zur Beurteilung der Wirksamkeit und Verträglichkeit des topischen Kombinationspräparates aus Mometasonfuroat 0,1\% und Salicylsäure 5\% im Rahmen der 1. Visite bei 3633 Patienten (99,5\%), im Rahmen der 2. Visite bei 3534 Patienten (96,8\%), im Rahmen der 3. Visite bei 3284 Patienten (89,9\%) und im Rahmen der Abschlussvisite bei $3433 \mathrm{~Pa}-$
Tab. 1 Demographische und klinische Patientendaten.

\begin{tabular}{|c|c|}
\hline Patientenanzahl & 3652 \\
\hline Anzahl Frauen (\%) & $1695(46,41 \%)$ \\
\hline Anzahl Männer (\%) & $1902(52,08 \%)$ \\
\hline Alter & 49,4 \pm 16 Jahre \\
\hline Behandlungsdauer & $41,0 \pm 23,3$ Tage \\
\hline \multicolumn{2}{|l|}{ Anzahl der Applikationen } \\
\hline $1 \times$ täglich & $1855(50,79 \%)$ \\
\hline $2 \times$ täglich & $1767(48,38 \%)$ \\
\hline unbekannt & $30(0,82 \%)$ \\
\hline \multicolumn{2}{|l|}{ Schweregrad } \\
\hline mittelschwere Psoriasis & $67,50 \%$ \\
\hline schwere Psoriasis & $19,20 \%$ \\
\hline \multicolumn{2}{|l|}{ Betroffene Körperfläche } \\
\hline$\leq 10 \%$ & $12,20 \%$ \\
\hline $10 \%-30 \%$ & $45,00 \%$ \\
\hline $30 \%-50 \%$ & $29,80 \%$ \\
\hline $50 \%-70 \%$ & $9,40 \%$ \\
\hline $90 \%-100 \%$ & $0,10 \%$ \\
\hline Anzahl der Visiten & 4 \\
\hline
\end{tabular}

tienten (94,0\%) erhoben. Im Anschluss erhielten 48,2\% der Patienten eine fortgesetzte Behandlung der Psoriasis mit einer Monotherapie. Am häufigsten wurden Glukokortikosteroide (20,4\% der Patienten) und Antipsoriatika (18,3\% der Patienten) eingesetzt.

\section{Ergebnisse \\ $\nabla$}

Wirksamkeit

Die Beurteilung der Wirksamkeit des topischen Kombinationspräparates aus Mometasonfuroat 0,1\% und Salicylsäure 5\% bei der mittelschweren und schweren Psoriasis beruhte auf einer Analyse der absoluten Veränderungen von Ausgangs- (1. Visite) bzw. Endwerten (Abschlussvisite) für die Schwere der Erkrankung, die Ausdehnung, die typischen klinischen Psoriasissymptome und die abschließende Globalbeurteilung durch Arzt und Patient unter Berücksichtigung der Applikationshäufigkeit.

Schwere der Psoriasis. Der klinische Status der Psoriasis wurde gemäß einer 4-Punkte-Skala mit kein (=0 Punkte), leicht (=1 Punkt), mittelschwer (= 2 Punkte) oder schwer (= 3 Punkte) eingestuft.

Der durchschnittliche Schweregrad zeigte im Beobachtungsintervall einen Rückgang von 2,1 $\pm 0,6$ auf $1,0 \pm 0,6$ Punkte $(\mathrm{p}<0,0001)$ ( Abb. 1a). Die Häufigkeit des im Rahmen der 1. Visite als mittelschwer (67,5\%) bzw. schwer (19,2\%) eingestuften Hautbefundes sank bei der Abschlussvisite auf 15,8\% bzw. 1,6\%. Insgesamt zeigte die Mehrzahl der Patienten bei der 4 . Visite keinen $(14,7 \%)$ oder nur noch einen leichten $(65,4 \%)$ Hautbefund.

Ausdehnung der Psoriasis. Bei jeder Visite erfolgte die Beurteilung der betroffenen Körperoberfläche nach einer Skala von 0 -3. Ein Wert von 1 bedeutete dabei eine betroffene Körperoberfläche $<10 \%$, ein Wert von 2 eine betroffene Körperoberfläche zwischen 10 und $30 \%$ und ein Wert von 3 zwischen 30 und $50 \%$.

Bei der Ausgangsvisite lag der Anteil der Patienten mit dem Skalenwert 1 für die betroffene Körperoberfläche bei zunächst $12,2 \%$, für den Skalenwert 2 bei 45,0\% und für den Skalenwert 3 bei $29,8 \%$. Bei der Abschlussvisite hingegen zeigten 12,4\% der Patienten keinerlei Symptome mehr (Skalenwert 0), 35,2\% der 


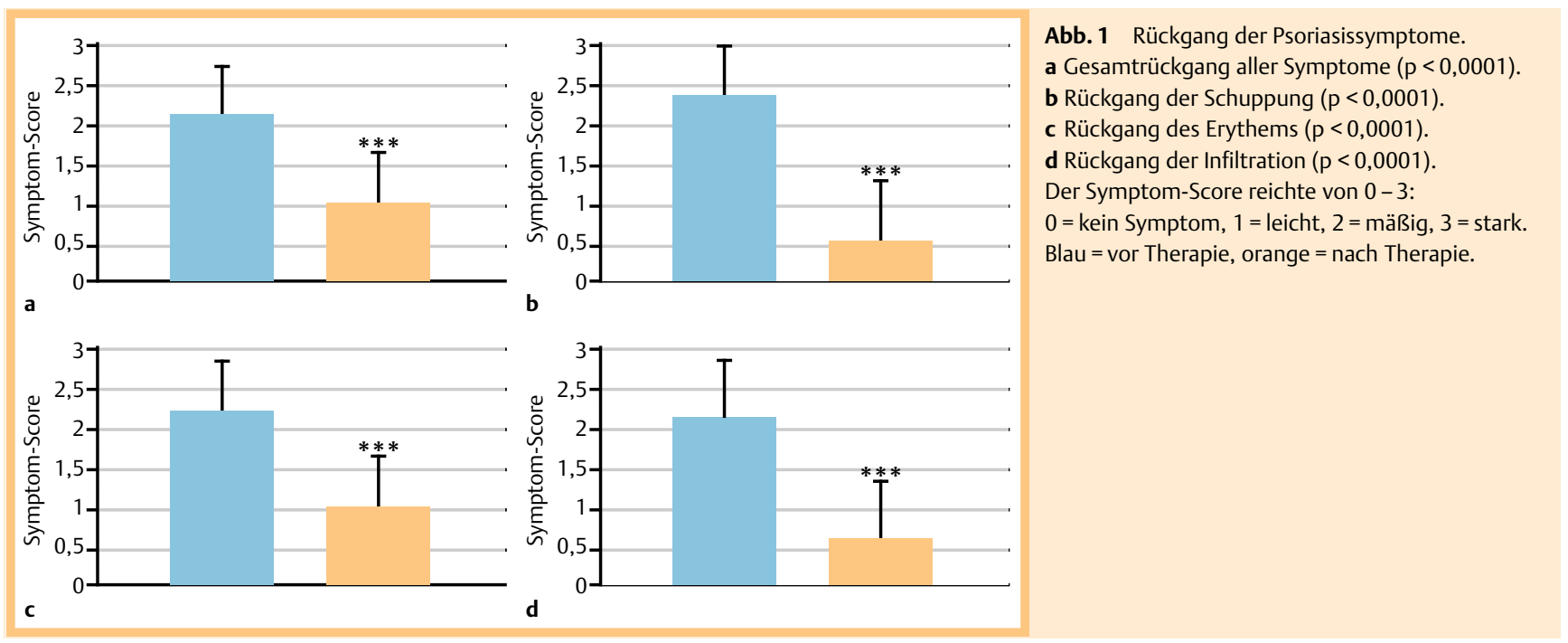

Patienten waren dem Skalenwert 1, 33,6\% der Patienten dem Skalenwert 2 und nur noch 10,8\% der Patienten dem Skalenwert 3 zuzuordnen.

Psoriasis-Symptome. Bei der Abschlussuntersuchung hatten sich darüber hinaus auch alle typischen klinischen Psoriasis-Symptome unter der Therapie mit dem topischen Kombinationspräparat aus Mometasonfuroat 0,1\% und Salicylsäure 5\% zurückgebildet: So zeigte sich für das Symptom Erythem im Beobachtungszeitraum eine durchschnittliche Verringerung der Ausprägung von $1,3 \pm 0,7$ ( $p<0,0001$ ). Während bei der 1 . Visite bei $54,1 \%$ der Patienten das Erythem als mittelgradig eingestuft wurde, zeigten bei der Abschlussvisite 60,3\% der Patienten nur noch ein leichtes Erythem. Auch für das Symptom Infiltration zeigte sich eine durchschnittliche Verringerung der Ausprägung von $1,5 \pm 0,8$ ( $p<0,0001$ ). Während bei der 1 . Visite bei $55,4 \%$ der Patienten die Infiltration noch als mittelgradig eingestuft wurde, zeigten bei der 4 . Visite bereits 45,9\% der Patienten keine Symptome mehr. Für das Symptom Schuppung zeigte sich eine durchschnittliche Verringerung der Ausprägung von sogar 1,9 $\pm 0,8$ ( $p<0,0001$ ). Während bei der 1 . Visite bei $45,8 \%$ der Patienten die Schuppung als mittelgradig eingestuft wurde, zeigten bei der 4. Visite 59,9\% der Patienten keine Symptome mehr ( $\triangle$ Abb. 1 b-d).

Globalbeurteilung. Ein weiterer Teil der Untersuchung berücksichtigte die Globalbeurteilung der Wirksamkeit des topischen Kombinationspräparates aus Mometasonfuroat 0,1\% und Salicylsäure $5 \%$. Diese spiegelte nochmals die oben gezeigte Verringerung der betroffenen Körperoberfläche und die Verbesserung der klinischen Psoriasis-Symptome wider. Im Einzelnen wurde die Wirksamkeit für 34,1\% der Patienten als exzellent (Verbesserung zwischen 75 und $<100 \%$ ) und für 35,4\% der Patienten als gut (Verbesserung von 50 bis $75 \%$ ) bezeichnet. 13,0\% der Patienten galten als geheilt. Auch wurde der globale subjektive Eindruck der Wirksamkeit des topischen Therapeutikums von 87,5\% der Ärzte und 84,9\% der Patienten als gut bis exzellent beurteilt ( $\bullet$ Abb. 2a,b).

\section{Anzahl der Applikationen}

Die Häufigkeit der Applikation des topischen Kombinationspräparates aus Mometasonfuroat 0,1\% und Salicylsäure 5\% betrug $1 \times$ täglich bei $58,8 \%$ der Patienten versus $2 \times$ täglich bei $48,4 \%$ der Patienten. Beide Patientengruppen waren bezüglich wichtiger klinischer Charakteristika (Erythem, Infiltration und Schup-

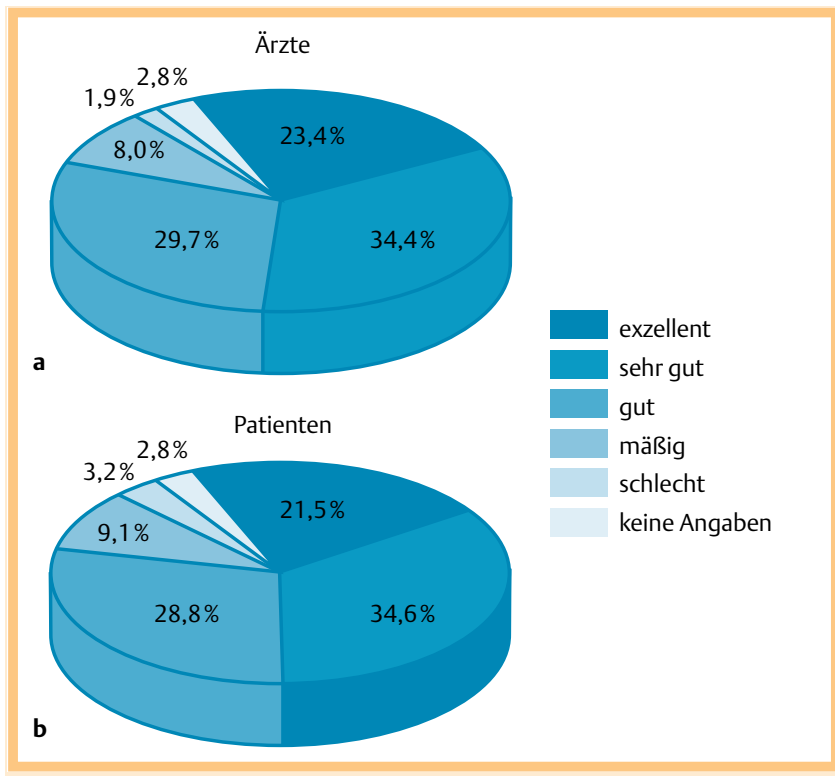

Abb. 2 Globalbeurteilung der Wirksamkeit.

pung) miteinander vergleichbar. Zwischen der Wirksamkeit einer $1 \times$ und $2 \times$ täglichen Applikation konnten zu keinem Zeitpunkt statistisch signifikante Unterschiede gefunden werden. Auch bei der Globalbeurteilung der Therapie mit dem topischen Kombinationspräparat ergaben sich zwischen beiden Gruppen keine signifikanten Unterschiede.

\section{Verträglichkeit}

Eine wichtige Anforderung, welche an ein modernes topisches Antipsoriatikum gestellt wird, bezieht sich auf seine gute Verträglichkeit und Sicherheit für den routinemäßigen Einsatz in der dermatologischen Praxis. 95,4\% der Ärzte und 94,1\% der Patienten bewerteten die Verträglichkeit des topischen Kombinationspräparates aus Mometasonfuroat 0,1\% und Salicylsäure $5 \%$ als gut bis exzellent. Die Häufigkeit einer mäßigen oder schlechten Verträglichkeit betrug $1,4 \%$ bei den Ärzten und weniger als $3 \%$ bei den Patienten ( $\bullet$ Abb. 3 a, b). Im Verlauf der Behandlung mit dem topischen Therapeutikum entwickelten 12 Patienten (0,33\%) insgesamt 16 unerwünschte Ereignisse. Bei 8 Patienten $(0,22 \%)$ traten nicht-schwerwiegende unerwünschte Ereignisse auf. Ein Patient hatte ein nicht-schwerwiegendes Er- 


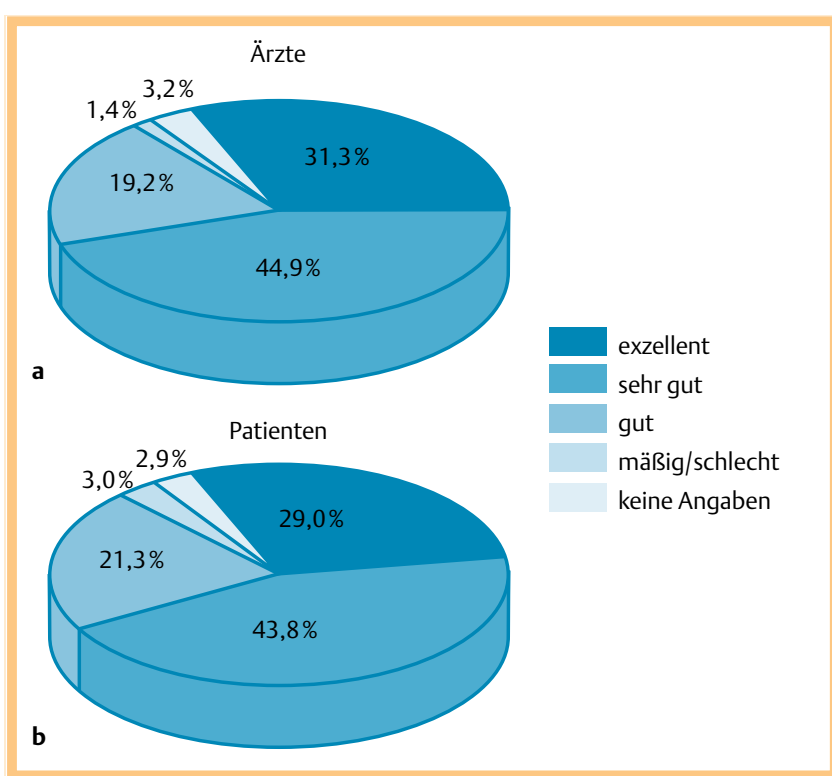

Abb. 3 Beurteilung der Verträglichkeit.

eignis, das in keinem Kausalzusammenhang mit der topischen Therapie stand. Bei den nicht-schwerwiegenden unerwünschten Ereignissen traten am häufigsten Juckreiz und Erythem auf. Bei 3 Patienten $(0,08 \%)$ wurden so genannte schwerwiegende unerwünschte Ereignisse rapportiert, die aus einer zum Zeitpunkt der statistischen Analyse ungeklärten Ursache zur Hospitalisierung der Patienten führten. Bei 3 unerwünschten Ereignissen wurde der Ausgang als wiederhergestellt dokumentiert, bei 2 als verbessert und bei 4 Ereignissen wurde der Ausgang nicht näher spezifiziert. Bei den anderen 7 Ereignissen erfolgte keine Verdachtsmeldung auf eine unerwünschte Arzneimittelwirkung, der Ausgang ist daher nicht bekannt.

\section{Compliance}

Eine schlechte Compliance war kein relevanter Punkt bei der Therapie mit dem topischen Kombinationspräparat aus Mometasonfuroat $0,1 \%$ und Salicylsäure $5 \%$. Vielmehr wurde die Compliance bei $42,9 \%$ der Ärzte und $43,0 \%$ der Patienten als sehr gut eingestuft. Die Häufigkeit von mäßiger oder fehlender Compliance im Zusammenhang mit der Behandlung lag in der Beurteilung durch Arzt und Patient unter 5\%.

\section{Diskussion}

$\nabla$

Anwendungsbeobachtungen von Arzneimitteln sind als nichtinterventionelles Beobachtungsinstrument zur Erfassung von Hauptwirkungen, aber auch unerwünschter Wirkungen von Arzneimitteln in einer breiten, nicht-selektierten Patientenpopulation besonders geeignet. Gerade bei neuen Arzneimitteln können adäquat dimensionierte Anwendungsbeobachtungen als Instrument der Pharmakovigilanz dazu beitragen, zusätzliche Sicherheitsdaten eines Präparates zu gewinnen. In der vorliegenden Anwendungsbeobachtung wurden unmittelbar nach Zulassung des neuartigen fixen Kombinationspräparates aus Mometasonfuroat 0,1\% und Salicylsäure 5\% über insgesamt 4 Wochen Daten bei 3652 Patienten mit mittelschwerer und schwerer Psoriasis erhoben.

Wie bereits in den kontrollierten Studien, konnte auch in der Praxisanwendung eine sehr gute Wirksamkeit des topischen
Kombinationspräparates aus Mometasonfuroat 0,1\% und Salicylsäure $5 \%$ dokumentiert werden. So wurden bemerkenswert gute Ergebnisse bei der Verbesserung des klinischen Status der Psoriasis beobachtet. Die Häufigkeit des bei Therapiebeginn als mittelschwer (67,5\%) bzw. schwer (19,2\%) eingestuften Hautbefundes sank bei Therapieende auf 15,8\% bzw. 1,6\%. Insgesamt zeigten bei der Abschlussuntersuchung 14,7\% der Patienten keinen Restbefund oder nur einen leichten $(65,4 \%)$ Hautbefund. Im Behandlungszeitraum verringerte sich auch die Ausdehnung der Psoriasisherde. So sank der Anteil der Patienten mit einer betroffenen Körperoberfläche < 10\% (Anteil: 12,2\%) bzw. zwischen 10 und 30\% (Anteil: 45,0\%) oder zwischen 30 und 50\% (Anteil: 29,8\%) der Körperoberfläche im Behandlungszeitraum stark ab: $12,4 \%$ der Patienten zeigten bei der Abschlussuntersuchung eine vollständige Remission, 35,2\% der Patienten waren der Kategorie mit einer betroffenen Körperoberfläche $<10 \%$ zuzuordnen, 33,6\% der Patienten der Kategorie mit einer betroffenen Körperoberfläche zwischen 10 und $30 \%$ und nur noch $10,8 \%$ der Patienten wiesen eine betroffene Körperoberfläche zwischen 30 und $50 \%$ auf. Diese insgesamt sehr gute Wirksamkeit des topischen Kombinationspräparates wurde im 4-wöchigen Beobachtungszeitraum auch durch eine deutliche Verbesserung bei den Einzelsymptomen Erythem, Infiltration und Schuppung sichtbar. Daher war auch der globale klinische Eindruck bei über $87 \%$ der Ärzte und über $84 \%$ der Patienten gut bis exzellent. Besonders hervorzuheben ist, dass hinsichtlich der Anzahl der Applikationen des topischen Therapeutikums kein statistisch signifikanter Unterschied zwischen einmal und zweimal täglicher Anwendung herausgestellt werden konnte.

Auch die Verträglichkeit des topischen Kombinationspräparates aus Mometasonfuroat 0,1\% und Salicylsäure 5\% kann nach den Daten der vorliegenden Anwendungsbeobachtung als sehr gut bezeichnet werden. Obwohl bei einem nennenswerten Teil der Patienten größere Hautflächen als 10\% über mehrere Wochen behandelt wurden, war keine Häufung von Nebenwirkungen zu verzeichnen. Nur bei 12 Patienten $(0,33 \%)$ traten unerwünschte Ereignisse auf. Die überwiegende Anzahl der gemeldeten Ereignisse war dabei unspezifischer Natur und von milder Ausprägung. Hinweise für ein gehäuftes Auftreten neuer, zum Zulassungszeitpunkt nicht bekannter Nebenwirkungen unter der Therapie mit dem topischen Kombinationspräparat ergaben sich nicht. Diese Ergebnisse fanden ihre Entsprechung in einer sehr guten globalen Beurteilung der Verträglichkeit durch Arzt und Patient.

Im Rahmen der vorliegenden Dokumentation konnte auch eine sehr hohe Patienten-compliance verzeichnet werden. Dies dürfte im Wesentlichen auf der ausgesprochen guten Wirksamkeit und Verträglichkeit des topischen Therapeutikums sowie der Zeitersparnis durch das einfache Behandlungsschema (1-2× tägliche Applikation) beruhen. Auch seitens der Ärzte wurde die Compliance als sehr gut bezeichnet.

Insgesamt konnte in der vorliegenden offenen, multizentrischen Anwendungsbeobachtung somit gezeigt werden, dass das topische Kombinationspräparat aus Mometasonfuroat 0,1\% und Salicylsäure 5\% auch im Praxisalltag eine sehr sichere und wirksame Therapie der mittelschweren und schweren Psoriasis darstellt. In diesem Zusammenhang ist es wichtig zu betonen, dass etwa $20 \%$ der mehr als 2 Millionen in Deutschland betroffenen Psoriasis-Patienten eine mittelschwere bis schwere Ausprägung ihrer Erkrankung aufweisen, wobei die starke Plaquebildung die Lebensqualität der Patienten erheblich beeinträchtigt [1,6]. Zur topischen Therapie der Psoriasis sollten stark antientzündlich 
wirksame Glukokortikosteroide mit sehr guter Hautverträglichkeit zum Einsatz kommen. Nach den Empfehlungen der Deutschen Dermatologischen Gesellschaft ist die hohe antiphlogistische Potenz von Mometasonfuroat, einem Glukokortikosteroid der Wirkstärke III, zur Behandlung der Psoriasis indiziert [5]. Zusätzlich zur Entzündungshemmung benötigt der Psoriatiker jedoch eine Substanz, die zu einer Verbesserung der hyperkeratotischen Komponente der Erkrankung führt. Eine effiziente Schuppenlösung wird mit 5\%iger Salicylsäure erreicht $[1,10]$. Dem Patienten steht nun erstmals eine fixe Kombination dieser beiden Substanzen zur Verfügung, welche die zweifachen, antientzündlichen und keratolytischen Wirkprinzipien der Psoriasistherapie in einer Salbe vereint und durch ihre synergistische Wirkung zu einem schnellen und sehr guten Therapieerfolg führt.

Wie zahlreiche Studien belegen, verbindet gerade Mometasonfuroat in besonderer Weise eine gute Wirksamkeit mit einer hohen Sicherheit, da nur vergleichsweise selten Symptome einer Hautatrophie beobachtet werden und systemische Effekte durch Beeinflussung der Hypothalamus-Hypophysen-Nebennierenrinden-Achse bei sachgemäßer Anwendung nicht zu erwarten sind. Auch ist das allergene Potenzial als gering einzustufen [5,11-13]. Zudem haben frühere Studien bereits eindrücklich dokumentiert, dass Mometasonfuroat $0,1 \%$ bei einer $1 \times$ täglichen Behandlung der Psoriasis über 2 bis 8 Wochen wirksamer ist als die meisten Steroide gleicher oder geringerer Potenz, wie Betamethasonvalerat 0,1\%, Fluocinolonacetonid 0,025\%, Fluticasonpropionat $0,005 \%$ und andere $[5,14]$. Aufgrund des in der vorliegenden Anwendungsbeobachtung gezeigten, besonders günstigen Sicherheitsprofils der fixen Kombination aus Mometasonfuroat und Salicylsäure ist dieses Therapeutikum im Schub über mehrere Wochen problemlos anzuwenden.

\section{Fazit für die Praxis}

\section{$\nabla$}

Gemäß der vorliegenden Beobachtungen unter Praxisbedingungen bietet das topische Kombinationspräparat aus Mometasonfuroat $0,1 \%$ und Salicylsäure $5 \%$ eine sehr gute Wirksamkeit, Verträglichkeit und Sicherheit für die Behandlung der mittelschweren bis schweren Psoriasis. Die hervorragende Verträglichkeit, die neuartige Galenik der Salbe und die einfache, 1-2 $\times$ tägliche Applikation haben wesentlichen Einfluss auf die dokumentierte hohe Compliance und leisten einen wichtigen Beitrag zur verbesserten Lebensqualität des Psoriasis-Patienten. Neben einer Monotherapie mit dem topischen Therapeutikum sind auch von der kombinierten Anwendung mit anderen Therapiemodalitäten gute Erfolge bei der Behandlung der Psoriasis zu erwarten.

\section{Abstract}

\section{Multicenter Observational Study of Mometasone} Furoate $0.1 \%$ - Salicylic Acid 5\% Ointment in Topical Treatment of Psoriasis Patients

$\nabla$

In a multicenter observational study the effectiveness, safety and tolerability of a mometasone furoate $0.1 \%$ - salicylic acid $5 \%$ ointment in topical treatment of 3652 patients with moderate to severe psoriasis was assessed. During 4 weeks of topical treatment all clinical symptoms of psoriasis improved. Global clinical impression was good to excellent in more than $87 \%$ of patients. In line, more than $84 \%$ of patients were at least satisfied with the combined topical treatment. Less than $0.4 \%$ of patients reported minor side effects. Taken together, these findings support the safety and efficacy of topical treatment with mometasone furoate $0.1 \%$ - salicylic acid $5 \%$ ointment, and suggest that this well-tolerated fixed combination is a useful addition to topical therapy of psoriasis patients in everyday practice.

\section{Literatur}

1 Gerdes S, Mrowietz U. Klassische Therapien der topischen Psoriasisbehandlung. Hautarzt 2006; 57: 666-671

2 Griffiths CE, Barker JN. Pathogenesis and clinical features of psoriasis. Lancet 2007; 370: 263-271

3 Menter A, Griffiths CE. Current and future management of psoriasis. Lancet 2007; 370: $272-284$

4 Naldi L, Gambini $D$. The clinical spectrum of psoriasis. Clin Dermatol 2007; 25: $510-518$

5 Nast A, Kopp I, Augustin M et al. German evidence-based guidelines for the treatment of psoriasis vulgaris (short version). Arch Dermatol Res 2007; 299: $111-138$

6 Weinstein GD, Krueger JD. Overview of psoriasis. In: Weinstein GD, Gottlieb AB (Hrsg). Therapy of moderate to severe psoriasis. Portland: National Psoriasis Foundation, 1994: 1 - 22

7 Koo J, Cuffie CA, Tanner DJ et al. Mometasone furoate $0.1 \%$ - salicylic acid $5 \%$ ointment versus mometasone furoate $0.1 \%$ ointment in the treatment of moderate to severe psoriasis: a multicenter study. Clin Ther 1998; 20: 283 - 291

8 Prakash A, Benfield P. Topical mometasone. A review of its pharmacological properties and therapeutic use in the treatment of dermatological disorders. Drugs 1998; 55: 145- 163

9 Medansky RS, Cuffie CA, Tanner DJ. Mometasone furoate $0.1 \%$ - salicylic acid $5 \%$ ointment twice daily versus fluocinonide $0.05 \%$ ointment twice daily in the management of patients with psoriasis. Clin Ther 1997; 19: 701 - 709

10 Stein GoldL, Corvari $L$. The roles of safety and compliance in determining effectiveness of topical therapy for psoriasis. Cutis 2007; 79: $32-38$

$11 \mathrm{Katz}$ HI, Prawer SE, Watson MJ et al. Mometasone furoate ointment $0.1 \%$ versus hydrocortisone ointment $1.0 \%$ in psoriasis. Atrophogenic potential. Int J Dermatol 1989; 28: 342 - 344

12 Kelly JW, Cains GD, Rallings $M$ et al. Safety and efficacy of mometasone furoate cream in the treatment of steroid responsive dermatoses. Australas J Dermatol 1991; 32: 85-91

13 Michel G, Nowok K, Beetz A et al. Novel steroid derivative modulates gene expression of cytokines and growth regulators. Skin Pharmacol 1995; 8: $215-220$

14 Swinehart JM, Barkoff JR, Dvorkin D et al. Mometasone furoate lotion once daily versus triamcinolone acetonide lotion twice daily in psoriasis. Int J Dermatol 1989; 28: 680-681 